\title{
OPTIMIZING FARES AND TRANSFER DISCOUNTS FOR A BUS-SUBWAY CORRIDOR
}

\author{
Bing-Zheng $\mathrm{LIU}^{1}$, Ying-En GE ${ }^{2 *}$, Kai $\mathrm{CAO}^{3}, \mathrm{Xi} \mathrm{JIANG}^{4}$, Linyun $\mathrm{MENG}^{5}$ \\ ${ }^{1}$ School of Transportation and Logistics, Faculty of Infrastructure Engineering, \\ Dalian University of Technology, China \\ ${ }^{2}$ College of Transport and Communications, Shanghai Maritime University, China \\ ${ }^{1,3}$ School of Transportation and Vehicle Engineering, Shandong University of Technology, China \\ 4, 5 State Key Laboratory of Rail Traffic Control and Safety, Beijing Jiaotong University, China
}

Received 5 February 2016; revised 26 March 2016; accepted 6 April 2017

\begin{abstract}
This paper aims to optimize fares and transfer discounts for public transit service along a bus-subway corridor with the consideration of effects of uncertainty in travel times and difference in stop spacing between bus and subway services on passenger behavior. The former factor is captured by the reserved time in travel cost and the latter one produces some passenger Origin-Destination (O-D) pairs along the corridor that can not be served by one mode only. This problem is formulated as a bi-level program, of which the upper level maximizes the social welfare and the lower-level capturing traveler choice behavior is a variable-demand Stochastic User Equilibrium (SUE) assignment model. A Genetic Algorithm (GA) is applied to solve the bi-level program while the Method of Successive Averages (MSA) is adopted to solve the lower-level model. A series of numerical experiments are carried out to illustrate the performance of the model and solution method. Numerical results indicate that the implementation of transfer discounts may be of great benefit to the social welfare and that the uncertainty in travel time and the difference in stop spacing play an important role in determining optimal fares and transfer discounts for the service along a bus-subway corridor.
\end{abstract}

Keywords: transfer discount, uncertainty in travel time, stop distance, bi-level program, public transit corridor.

\section{Introduction}

In the past decade, a public transit system composed of bus and subway services has been advocated as an efficient and preferred way to meet ever-increasing urban travel demand and relieve traffic congestion in metropolitan cities around the world. The comprehensive effectiveness of such a combined transit system depends not merely on a reasonable design of its topology and timetable but also on an optimal transit fare structure for it, including fares and transfer discounts. As a flexible instrument, the fare structure may influence passenger behavior directly and the operator' revenue ultimately. It is widely acknowledged that the higher fare may lead to the lower ridership. On the other hand, the low fare may result in a high subsidy from the government if the service sustains. In addition, the transfer pricing policy can also have a significant influence over passenger behavior. Therefore, optimizing fares and discounts of transfer fees between different lines and different services is a fundamental problem for the author- ity or operator to run a public transit system. This paper considers a combined bus and subway service in parallel along a traffic corridor, which is called a bus-subway corridor hereafter, and aims to find the optimal fare structure for the corridor given the travel demand and timetable.

The optimization of transit fare structure is well investigated in the literature on transportation economics (Kocur, Hendrickson 1982; Yang, Kin 2000) and network equilibrium (Lam, Zhou 2000; Zhou et al. 2005; Watling 2006). The relevant research may be traced back to the 1970s, such as Nash (1978) and Glaister and Collings (1978), which proposed to treat the setting of a fare structure as an optimization problem. Originally, Nash (1978) and Glaister and Collings (1978) respectively used an elasticity based function and a linear demand function to capture the influence of travel costs on passenger behavior without regard to externalities (e.g. congestion). Spiess and Florian (1989) proposed a new assignment model for

\footnotetext{
${ }^{*}$ Corresponding author. E-mail: yege@shmtu.edu.cn

\#Associate Editor of the TRANSPORT - the manuscript was handled by one of the Editors, who made all decisions related to the manuscript (including the choice of referees and the ultimate decision on the revision and publishing).
} 
transit networks as an alternative way to find optimal strategies for public transit service. Li et al. (2009b) considered the optimal transit fare structure under different market regimes with uncertainty in a network. Tirachini et al. (2014) concerned multimodal pricing and optimal design of urban public transport with a focus on the interplay between traffic congestion and bus crowding. Kaddoura et al. (2015) found a range of values for the optimal fare and headway, due to the randomness in user behavior that is inherent to an agent-based approach. De Palma et al. (2015) derived the optimal time-table and the optimal prices considering crowding in public transport and its implications for pricing, seating capacity choice and optimal scheduling. There is also a great deal of effort put into consideration of capacity constraints, dwell time, fare collection systems, etc. (Tirachini, Hensher 2011; Tang et al. 2016). Some work in the literature focuses on the issue of coordinated pricing for different modes in a city. Li et al. (2009a) optimized a bus-rail transit system with feeder bus services under different market regimes. Wang et al. (2014) considered demand uncertainty and bounded rationality in optimizing the transit fare in an urban transportation system with public transit service and private cars. Lu et al. (2015) enhanced the insights into pricing mechanism for subway and parking and corresponding mode choice behavior along the corridor with elastic demand. Tang et al. (2017) offered an approach to integrated optimization of bus line fare and operational strategies in a public transit corridor with elastic demand and Tang et al. (2019) optimized limited-stop bus services with a differential fare structure, which is carried out in a public transit corridor with bus service only. However, there is a paucity of the optimization of the fare structure that includes fares and transfer discounts of the services offered in a bus-subway corridor. Moreover, these existing investigations only considered the passenger Origin-Destination (O-D) pairs that can be served or covered by either bus or subway services directly. Generally, the stop spacing is the distance between two successive stops along a transit service line. Actually, the stop spacing often differs for bus and subway services along a combined bus-subway corridor. It means that there are some added groups of passenger O-D pairs that can not be served only by one of the two services directly. These pairs can be served by a combined bus-subway service with transfer. Thus, the difference in stop spacing of the two services should be taken into account in optimizing fares and transfer discounts for a bus-subway corridor.

In addition, the travel time of either of the two transit modes is normally uncertain and variable due to congestion and other random factors. In order to analyze passenger choice behavior under stochastic travel times, Noland and Polak (2002) and Shao et al. (2006) built a logit model based on the random utility theory. Fujii and Kitamura (2004) and Avineri (2006) integrated the travel behavior mechanism and the cumulative prospect theory to simu- late passenger behavior under varied uncertain factors. Yao et al. (2014) presented a transit network optimization method, in which the travel time reliability on the road was considered. A robust optimization model was formulated to take into account reliable transit service with stochastic travel time. Yao et al. (2015) formulated generalized route travel costs with the uncertainty of link travel time and the uncertainty of waiting time at a bus stop and in-vehicle congestion costs for the bus mode. Ehrgott et al. (2015) and Chen et al. (2017) considered the travel time uncertainty faced by travellers when choosing among alternative routes. Due to the distinct operational circumstances of bus and subway services, the levels of influence due to uncertainty may differ significantly between the two services. Thus, this paper also considers the influence of uncertainty in travel time of mode on passenger behavior and transforms it into an objective component of the travel cost by defining it as a reserved time in trip, just as the uncertainty effects on a transit network are measured by a function of the standard deviation of travel times on transit links in Li et al. (2009b).

The optimization of fare structure can be based on different objectives (Li et al. 2009b; Borndörfer et al. 2012; Tang et al. 2016, etc.). Whatever the forms of an objective are, the passengers' response to the fare pricing is the key part in all these investigations and is captured by means of a multinomial logit-based Stochastic User Equilibrium (SUE) model. However, the multinomial logit model has a drawback in dealing with the "common segment" of alternative paths. To avoid this, we choose the Path-Size logit model, for further details of which the reader may refer to Frejinger and Bierlaire (2007) and Ramming (2002).

The key aim of this work is to confirm advantages of optimizing the whole fare structure, especially transfer discounts, along a combined bus-subway service corridor. The other aim is to analyse impacts of consideration of new passenger O-D pairs and uncertainty in travel time of mode on the optimal fare structure. The two intellectual merits differentiate this work from Liu et al. (2017). Another intellectual merit of this work is the integration into the optimization model of difference in stop spacing between bus and subway services and the influence of uncertainty in travel time of each mode on passenger behavior. The demand along such a corridor is served by bus service only, subway service only or their conbined service. Therefore, the O-D pairs along the corridor can be classified into three types according the transit service they receive.

Following this introduction, Section 1 describes the representation of a bus and subway corridor and lists basic assumptions of this investigation. Section 2 defines the components of the travel cost that impact passenger behavior. Section 3 presents model formulation and solution algorithm. Section 4 provides a set of numerical experiments to illustrate the applications of the proposed models. This paper closes with some concluding remarks. 


\section{Preliminaries}

\subsection{Assumptions}

The paper considers a corridor $G(N, L)$ composed of a bus line and a subway line, as shown schematically in Figure 1 , where $N$ is the set of nodes $n$ and $L$ is the set of segments connecting each pair of adjacent nodes. Let $W$ be the set of O-D pairs, $w$ represent an O-D pair, and $M$ be the set of paths for passengers. The bus line serves all $\mathrm{O}-\mathrm{D}$ pairs along the corridor, and the subway only can serve part of the O-D pairs, because the gaps between two subway stops are usually larger than those for the bus service. In addition, there is usually a walking distance for passengers to transfer at a stop when this transfer takes place from the bus line to subway or vice versa.

Before proceeding, let us make some basic assumptions as follows:

- transit line - the distance between two successive stations of the subway line is twice as much as for the bus line. Each service has a constant frequency, and the average vehicle operating speed of each service is given and invariable;

- passenger - all passengers are assumed to be homogeneous, i.e., they have an identical value of time. There are four alternative paths for a passenger to complete her/his travel, namely, bus direct path, subway direct path and mode-combined. With regard to transfers, passengers are also assumed never to use one mode for twice in a trip, which means they can transfer once only.

\subsection{Classification of $\mathrm{O}-\mathrm{D}$ pairs}

According to Section 1.1, transit O-D pairs in the bus and subway corridor are classified into three types in terms of their alternative paths as shown in Table 1.

Table 1. Classification of O-D pairs

\begin{tabular}{|c|c|c|c|c|c|}
\hline \multirow{2}{*}{$\begin{array}{l}\text { O-D } \\
\text { pairs }\end{array}$} & \multirow{2}{*}{$\begin{array}{c}\text { Transfer } \\
\text { node }\end{array}$} & Origin & Destination & \multirow{2}{*}{$\begin{array}{c}\text { Direct } \\
\text { path }\end{array}$} & \multirow{2}{*}{$\begin{array}{c}\text { Transfer } \\
\text { path }\end{array}$} \\
\hline & & $\begin{array}{c}\text { Subway } \\
\text { line }\end{array}$ & $\begin{array}{l}\text { Subway } \\
\text { line }\end{array}$ & & \\
\hline \multirow[t]{2}{*}{$\begin{array}{l}\text { Type } \\
\text { one }\end{array}$} & yes & yes & yes & $\begin{array}{l}\text { bus, } \\
\text { subway }\end{array}$ & $\begin{array}{l}\text { bus-subway, } \\
\text { subway-bus }\end{array}$ \\
\hline & no & yes & yes & $\begin{array}{l}\text { bus, } \\
\text { subway }\end{array}$ & - \\
\hline $\begin{array}{l}\text { Type } \\
\text { two }\end{array}$ & no & no & no & bus & - \\
\hline \multirow{3}{*}{$\begin{array}{l}\text { Type } \\
\text { three }\end{array}$} & \multirow[t]{2}{*}{ yes } & yes & no & bus & subway-bus \\
\hline & & no & yes & bus & bus-subway \\
\hline & no & no & no & bus & - \\
\hline
\end{tabular}

Type one is those $\mathrm{O}-\mathrm{D}$ pairs of which both the origin and destination nodes are served by both bus and subway lines, e.g. O-D pair $(n, n+4)$ and $(n, n+2)$ in Figure 1 . They can complete their trips by four alternative paths, namely, bus direct path, subway direct path, combined bus-subway transfer path or combined subway-bus transfer path if only one transfer is allowed.

Type two is those O-D pairs which are served directly by bus service, but no direct subway service whereas the combined service is not a seasonable choice, e.g. O-D pair $(n+1, n+2)$ and $(n+2, n+3)$ in Figure 1 .

Type three is those O-D pairs of which both the origin and destination nodes are served by bus lines while only one of the end nodes is served by subway service, e.g. O-D pair $(n, n+3)$ and $(n+1, n+4)$ in Figure 1. In addition to the bus direct path, a passenger between an $\mathrm{O}-\mathrm{D}$ pair of this type may take an alternative combined transfer path (bus-subway or subway-bus transfer path, dependent on which end node of the O-D pair is connected to the subway service).

Contrasting with the existing literature, types two and three of O-D pairs are termed added O-D pairs. It is noteworthy that only type one of O-D pairs exist if the stop gaps for bus and subway services are the same and both services share their stops.

\section{Travel cost}

Transit passengers make their path choice decision based on the travel costs of all alternatives. There are several basic components in the travel cost, e.g. walking time, waiting time, in-vehicle travel time, in-vehicle crowding discomfort, reserved time, fares and/or transfer cost, which are respectively defined as follows.

Walking time. This is the time it takes a passenger to enter and leave a subway station. This part of the journey is mainly done on foot or by walking although the lift or elevator may be used from time to time. Therefore, we term the part of journey time walking time. The walking time at a bus station $T w$ is disregarded here. In addition, a transit passenger also needs some time, $T T r$, to stations between two service lines.

Waiting time. The average waiting time $T W_{m}$ a passenger spends at a station of transit mode $m(b=$ bus, $s=$ subway) can be calculated by:

$$
T W_{m}=\frac{\gamma_{m}}{f_{m}}, m \in\{b, s\},
$$

where: $f_{m}$ is the service frequency of mode $m$; the parameter $\gamma_{m}$ depends on the distribution of transit vehicle headway and passenger arrival time. With an assumption

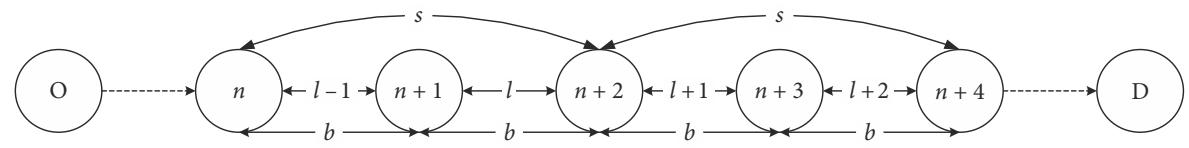

Figure 1. A bus and subway corridor 
of a uniform distribution of passenger arrival and a constant transit vehicle headway, the value of $\gamma_{m}$ is set to 0.5 (Lam, Morrall 1982).

In-vehicle travel time The average in-vehicle travel time $T_{m}^{L^{\prime}}$ by mode $m$ can be calculated in the following manner:

$$
T_{m}^{L^{\prime}}=\sum_{l_{i} \in L^{\prime}} \frac{d_{l_{i}}}{V_{m}}, m \in\{b, s\},
$$

where: $L^{\prime}$ is the set of travel segments, $l_{i} \in L^{\prime} ; d_{l_{i}}$ is the length of each segment; $V_{m}$ is the average vehicle operating speed of mode $m$.

In-vehicle crowding discomfort. According to Spiess and Florian (1989), the in-vehicle crowding discomfort cost is measured in terms of generalized time units, and can be expressed in the form of Bureau of Public Roads (BPR) type function with regard to the mean vehicle travel time, passenger volume, and vehicle capacity on the line. Considering the different automobile structure of two modes, an analogical uniform type function is applied to measure the cost of in-vehicle crowding discomfort $U C_{m}^{l}$ in mode $m$ through segment $l$, which can be expressed as:

$$
\begin{aligned}
& U C_{m}^{l}=\left(\eta_{m}^{0}+\eta_{m}^{1} \cdot\left(\max \left(0, v_{m}^{l}-f_{m} \cdot k_{m}\right)\right)\right) \cdot T_{m}^{l}, \\
& m \in\{b, s\},
\end{aligned}
$$

where: $\eta_{m}^{0}$ reflects the baseline discomfort level of mode $m$ through segment $l$ when the vehicle is empty; $\eta_{m}^{1}$ is the positive calibrated parameter of the in-vehicle discomfort function; $v_{m}^{l}$ is the passenger flow using mode $m$ on segment $l ; k_{m}$ is the vehicle capacity of mode $m$; $T_{m}^{l}$ is the in-vehicle travel time by mode $m$ on segment $l$.

Reserved time. Transit passengers may suffer from the delay caused by the uncertainty attributed to the operating environment of a transit mode or random factors. For the same transit mode, if the travel distance has a greater length, it is more difficult for a passenger to master the uncertainty level, which means the passenger may bear more delay. For a certain travel length, if the transit mode chosen by a passenger is easier to be interfered by uncertainty the passenger may bear more delay. Considering the uncertainty that may appear during passengers' trip, they have a rough measure of the travel time (in-vehicle travel time) prepared for trips. Normally, the difference between the rough measure and the actual measure of in-vehicle travel time is defined as a reserved time in passenger's trip. In the analysis, the reserved time $T R_{m}^{L^{\prime}}$, is captured by a function with regard to the in-vehicle travel time $T_{m}^{L^{\prime}}$, on the set of segments $L^{\prime}$, by mode $m$, i.e.:

$$
\begin{aligned}
& T_{m}^{L^{\prime}}=\left(\rho_{m}-1\right) \cdot T_{m}^{L^{\prime}}, \\
& m \in\{b, s\},
\end{aligned}
$$

where: $\rho_{m}(>1)$ is the parameter used to roughly measure the delay of mode $m$, from which is suffered by a passenger.

The travel cost $U_{M}^{w}$ of direct path $(s, b)$ for each passenger between an $\mathrm{O}-\mathrm{D}$ pair $w$ can be expressed as:

$$
\begin{aligned}
& U_{M}^{w}= \begin{cases}u_{1}, & M=s ; \\
u_{2}, & M=b,\end{cases} \\
& \forall w \in W,
\end{aligned}
$$

where:

$$
\begin{aligned}
& u_{1}=\alpha_{1} \cdot T w+\alpha_{2} \cdot T W_{s}+\alpha_{3} \cdot T_{s}^{L^{w}}+ \\
& \alpha_{4} \cdot p_{s}+\alpha_{5} \cdot T R_{s}^{L^{w}}+\alpha_{3} \cdot \sum_{l \in L^{w}} U C_{s}^{l} ; \\
& u_{2}=\alpha_{2} \cdot T W_{b}+\alpha_{3} \cdot T_{b}^{L^{w}}+\alpha_{4} \cdot p_{b}+ \\
& \alpha_{5} \cdot T R_{b}^{L^{w}}+\alpha_{3} \cdot \sum_{l \in L^{w}} U C_{b}^{l},
\end{aligned}
$$

where: the coefficients $\alpha$ are the reciprocal substitution factors between each cost component that is used to convert different quantities to the same unit. In this paper we set $\alpha_{3}$ to $1.0 ; \alpha_{1}$ is the ratio of the value of walking time to the value of in-vehicle travel time, $\alpha_{2}$ is the ratio of the value of waiting time to the value of in-vehicle travel time, $\alpha_{4}$ is the reciprocal of the value of in-vehicle travel time, and $\alpha_{5}$ is the ratio of the value of reserved time to the value of in-vehicle travel time. Besides, $L^{w}$ is the set of segments between O-D pair $w$; $T_{s}^{L^{w}}$ and $T_{b}^{L^{w}}$ are the in-vehicle travel times of the subway direct path and bus direct path, respectively; $T R_{s}^{L^{w}}$ and $T R_{b}^{L^{w}}$ are the corresponding reserved times for each direct path, respectively; $p_{s}$ and $p_{b}$ are the fares of subway and bus services, respectively.

It is assumed that, there are two transfer paths: bussubway $c_{1}$, and subway-bus $c_{2}$, whose travel cost $U_{M}^{w}$, for each passenger between an $\mathrm{O}-\mathrm{D}$ pair $w$ can be expressed as follows:

$$
\begin{aligned}
& U_{M}^{w}=\alpha_{1} \cdot(T w+T T r)+ \\
& \alpha_{2} \cdot\left(T W_{s}+T W_{b}\right)+T P+ \begin{cases}u_{1}, & M=c_{1} ; \\
u_{2}, & M=c_{2},\end{cases} \\
& \forall w \in W,
\end{aligned}
$$

where:

$$
\begin{aligned}
& u_{1}=\alpha_{3} \cdot\left(\sum_{l \in L_{c 1, s}^{W}} U C_{s}^{l}+\sum_{l \in L_{c 1, b}^{w}} U C_{b}^{l}\right)+ \\
& \alpha_{3} \cdot\left(T_{s}^{L_{c 1, s}^{W}}+T_{b}^{L_{c 1, b}^{w}}\right)+\alpha_{5} \cdot\left(T R_{s}^{L_{c 1, s}^{w}}+T R_{b}^{L_{c 1, b}^{w}}\right)+ \\
& \alpha_{4} \cdot\left(p_{b}+\lambda_{s} \cdot p_{s}\right) ; \\
& u_{2}=\alpha_{3} \cdot\left(\sum_{l \in L_{c 2, s}^{w}} U C_{s}^{l}+\sum_{l \in L_{c 2, b}^{W}} U C_{b}^{l}\right)+ \\
& \alpha_{3} \cdot\left(T_{s}^{L_{c 2, s}^{W}}+T_{b}^{L_{c 2, b}^{w}}\right)+\alpha_{5} \cdot\left(T R_{s}^{L_{c 2, s}^{W}}+T R_{b}^{L_{c 2, b}^{w}}\right)+ \\
& \alpha_{4} \cdot\left(p_{s}+\lambda_{b} \cdot p_{b}\right),
\end{aligned}
$$

where: the term $T P$ is the transfer penalty, which accounts for the resistance a passenger suffers from changing the service in addition to the walking time (Tong, Wong 1999); $L_{c 1, b}^{w}, L_{c 1, s}^{w}$ and $L_{c 2, b}^{w}, L_{c 2, s}^{w}$ are the sets of bus line segments and subway line segments in each transfer, respectively; $\lambda_{b}$ and $\lambda_{s}$ are the transfer discounts of the two services, respectively. 


\section{Model formulation}

The problem refers to the situation where the whole bus and subway corridor is operated by the single operator with an objective to maximize the total social welfare of the transit system. As such, the transit operator determines the fares and transfer discounts of the two services. Furthermore, the operator's decision must be influenced by the passenger behavior, and the operator should also seek for the reasonable decision on the fare structure in order to attract more passengers. For this purpose, the model to be proposed includes two levels: the upper level aims to maximize the social welfare and the lower-level is a variable-demand SUE assignment model.

\subsection{Upper sub-model}

The objective of social welfare includes consumer surplus and producer profit. Although the subsidy can be provided by the authority, it is not considered in the paper.

The consumer surplus is the total benefits all consumers obtain from exchange, which can be represented by $\sum_{w \in W} \frac{g_{w}}{\beta}$, measured in time units following Williams (1977) and Evans (1987). The parameter $\beta(>0)$ reflects the demand sensitivity to the expected travel cost. $g_{w}$ is the total resultant passenger demand between O-D pair $w$, which is assumed to be elastic and is specified as a negative exponential function with respect to the expected travel cost $E_{w}$, i.e.:,

$$
\begin{aligned}
& g_{w}=g_{w}^{0} \cdot \exp \left(-\beta \cdot E_{w}\right), \\
& \forall w \in W,
\end{aligned}
$$

where: $g_{w}^{0}$ is the initial potential passenger demand between $\mathrm{O}-\mathrm{D}$ pair $w$; according to the random utility theory, the expected travel cost $E_{w}$ can be measured by the following formula (Sheffi 1985; Ben-Akiva, Lerman 1985; Oppenheim 1995):

$$
\begin{aligned}
& E_{w}=-\frac{1}{\theta} \cdot \ln \left(\sum_{M} \exp \left(-\theta \cdot U_{M}^{w}\right)\right), \\
& M \in\left\{b, s, c_{1}, c_{2}\right\},
\end{aligned}
$$

where: the parameter $\theta(>0)$ describes the variation of passenger perception on travel cost in the path choice decision. In accordance with the discrete choice theory, $\theta \geq \beta$ must hold (Ben-Akiva, Lerman 1985).

The producer profit $\Phi$ is the total revenue that is generated from passenger fares minus the total operating costs, which can be expressed as:

$$
\begin{aligned}
& \Phi=\sum_{w \in W}\left(h_{b}^{w} \cdot p_{b}+h_{s}^{w} \cdot p_{s}+h_{c_{1}}^{w} \cdot\left(p_{b}+\lambda_{s} \cdot p_{s}\right)+\right. \\
& \left.h_{c_{2}}^{w} \cdot\left(p_{s}+\lambda_{b} \cdot p_{b}\right)\right)-\left(R_{s} \cdot C_{s}+R_{b} \cdot C_{b}\right),
\end{aligned}
$$

where: $h_{b}^{w}, h_{s}^{w}, h_{c 1}^{w}$ and $h_{c 2}^{w}$ are, respectively, the passenger demand of bus direct path, subway direct path and two transfer paths for O-D pair $w ; C_{b}$ and $C_{s}$ are the operating costs per vehicle hour of bus and subway service, respectively; $R_{b}$ and $R_{s}$ are respectively the number of vehicles for bus and subway service, which can be given by:

$$
\begin{aligned}
& R_{b}=f_{b} \cdot C T_{b} ; \\
& R_{s}=f_{s} \cdot C T_{s},
\end{aligned}
$$

where: $C T_{b}$ and $C T_{s}$ are the cycle journey times for the bus and subway services, respectively.

Then the objective function of social welfare $S W$ can be represented as:

$$
\begin{gathered}
\sum_{w \in W} g_{w} \\
S W(\mathbf{p}, \lambda)=\frac{}{\alpha_{4} \cdot \beta}+ \\
\sum_{w \in W}\left(h_{b}^{w} \cdot p_{b}+h_{s}^{w} \cdot p_{s}+h_{c_{1}}^{w} \cdot\left(p_{b}+\lambda_{s} \cdot p_{s}\right)+\right. \\
\left.h_{c_{2}}^{w} \cdot\left(p_{s}+\lambda_{b} \cdot p_{b}\right)\right)-\left(R_{s} \cdot C_{s}+R_{b} \cdot C_{b}\right)
\end{gathered}
$$

where:

$$
\begin{aligned}
& p_{b} \geq 0, p_{s} \geq 0 ; \\
& 0 \leq \lambda_{b} \leq 1,0 \leq \lambda_{s} \leq 1 ;
\end{aligned}
$$
ables.

$(\mathbf{p}, \lambda)=\left(p_{b}, p_{s} ; \lambda_{b}, \lambda_{s}\right)$ is the vector of decision vari-

\subsection{Lower-level sub-model}

The lower-level sub-model reflects the passengers' response to the given fare structure. Considering the clash between the Independence from Irrelevant Alternative (IIA) property of multinomial logit formulation and the common segments among the four alternative paths in the paper, a Path-Size Logit model (Ben-Akiva, Lerman 1985) is applied here, in which the probability $P_{M}^{w}$ that path $M$ is chosen between $\mathrm{O}-\mathrm{D}$ pair $w$ is defined by:

$$
\begin{aligned}
& P_{M}^{w}=\frac{P S_{M}^{w} \cdot \exp \left(-\theta \cdot U_{M}^{w}\right)}{\sum_{M} P S_{M}^{w} \cdot \exp \left(-\theta \cdot U_{M}^{w}\right)}, \\
& \forall w \in W, M \in\left\{b, s, c_{1}, c_{2}\right\},
\end{aligned}
$$

where: $P S_{M}^{w}$ is the added term to the travel cost of alternative paths, which can be expressed by:

$$
\begin{aligned}
& P S_{M}^{w}=\sum_{l \in L_{M}}\left(\frac{t_{m}^{l}+u_{m}^{l}}{T_{M}^{w}+U C_{M}^{w}}\right) \cdot \frac{1}{\sum_{M} \delta_{l M}}, \\
& \forall w \in W, \quad m \in\{b, s\}, \quad M \in\left\{b, s, c_{1}, c_{2}\right\},
\end{aligned}
$$

where: $t_{m}^{l}$ and $u_{m}^{l}$ are respectively the in-vehicle travel time and in-vehicle crowding discomfort on segment $l$ of mode $m ; \delta_{l M}=1$ if line segment $l$ is on path $M$ between O-D pair $w$, and $\delta_{I M}=0$ otherwise; $T_{M}^{w}$ and $U C_{M}^{w}$ are the total in-vehicle travel time and in-vehicle crowding discomfort on path $M$, respectively; $L_{M}$ is the set of segments of each mode on path $M$.

Then, the passenger flow $h_{M}^{w}$ on path $M$ can be computed by: 


$$
\begin{aligned}
& h_{M}^{w}=g_{w} \cdot P_{M}^{w}, \\
& \forall w \in W, \quad M \in\left\{b, s, c_{1}, c_{2}\right\},
\end{aligned}
$$

where: $g_{w}$ can be obtianed by Equations (1) and (2). Hence, the passenger flow on segment $l$ of mode $m, v_{m}^{l}$, can be expressed by:

$$
\begin{aligned}
& v_{m}^{l}=\sum_{w \in W} \sum_{M} \varphi_{m, M}^{l} \cdot h_{M}^{w}, \\
& \forall l \in L, m \in\{b, s\}, \quad M \in\left\{b, s, c_{1}, c_{2}\right\},
\end{aligned}
$$

where: $\varphi_{m, M}^{l}$ is a binary variable that equals 1 if the segment $l$ of mode $m$ is on path $M$ and 0 if it is not.

\subsection{Solution algorithm}

Considering the complexity of the bilevel optimization problem, a Genetic Algorithm (GA) with double-point crossover is adopted to solve the bi-level program while the Method of Successive Averages (MSA) (Sheffi 1985) is selected to solve the lower-level model.

The following algorithm is used to solve the previously formulated bi-level model:

- step 1 - initialization. Choose the values of relevant parameters in GA, including the population size, the maximum generation $N G$, the probabilities of performing crossover and mutation; select the range of variables $p_{m}, \lambda_{m}$; save $n_{g}=1$ for the loop time;

- step 2 - computation. Calculate the travel cost of each path, the path choice probability and the path passenger flow for each chromosome; count the objective function of each individual chromosome $S W(i)$; identify the maximum and minimum objective function values of population saving as $S W_{\max }$ and $S W_{\text {min }}$; define the feature of each individual chromosome as $S W(i)-S W_{\min }$;

- step 3 - operation. Perform selection, reproduction, crossover and mutation procedures;

- step 4 -verification. Terminate the operation when the loop time reaches the maximum generation $(n=N G)$, and output the data. Otherwise, let $n_{g}=$ $n_{g}+1$ and go to step 2 .

Where the following MSA procedure is employed to complete the computation in the aforementioned step 2:

- step 1 - initialization. Perform a stochastic network loading procedure based on a set of initial travel cost $U$. Then generate a set of passenger flow on the four paths $H A$. Set $n=1$;

- step 2 - update. Set $U^{(n)}=U\left(H A^{(n)}\right)$;

- step 3 - direction finding. Perform a stochastic network loading procedure based on the current set of path travel costs $U^{n}$ and then yield an auxiliary passenger flow pattern $H B^{(n)}$;
- step 4 - move. Set the new flow pattern as $H A^{(n+1)}=H A^{(n)}+\left(\frac{1}{n}\right) \cdot\left(H B^{(n)}-H A^{(n)}\right) ;$

- step 5 - convergence criterion. If the convergence is attained, stop. Otherwise, set $n=n+1$ and go to step 2 .

\section{Numerical experiments and analysis}

This section is to present a series of numerical experiments to illustrate the application and performance of the proposed model in handling different scenarios.

\subsection{Data input}

The sample corridor is shown in Figure 2. It consists of 15 nodes, one bus line that serves all nodes, one subway line that serves part of the nodes, and a bi-directional passenger demand matrix given in Table 2. The O-D pairs in dash area of Table 2 are type one $\mathrm{O}-\mathrm{D}$ pairs. The distances between two successive stops of bus and subway lines are assumed to be 0.6 and $1.2 \mathrm{~km}$, respectively. Each service line has its own stations. The walking time at a subway station $T w$ is set to $0.06 \mathrm{~h}$. The walking distance to transfer is assumed to be uniform in the whole corridor and measured by transfer walking time $T T r$ equal to $0.1 \mathrm{~h}$. Other parameter values for the numerical experiments are given in Table 3. The values used for capacities, operating costs, average operating speeds and frequencies of vehicle for the two services are displayed in Table 4.

\subsection{Analysis of numerical results}

\subsubsection{Effects of reserved time on the optimal fares}

We first explore the effects of the uncertainty, which is measured roughly by passengers, reserved time in a trip, referring to type one $\mathrm{O}-\mathrm{D}$ pairs in Table 2. For this purpose, it is necessary to consider three scenarios, which are described as follows:

- first scenario: optimize fares of the bus and subway two services when the reserved time is unconsidered;

- second scenario: capture passenger flow shares among different paths under the condition that fares of bus and subway services inherited from the first scenario;

- third scenario: optimize fares of the two services considering the reserved trip.

None of the three scenarios involve the optimization of transfer discounts, which means the transfer discounts for the two services $\lambda_{m}$ equals $100 \%$. The results of the first example are displayed in Table 5. Considering the first and second scenarios, the fares of bus and subway services are the same in the two scenarios. With the same travel length, the presence of reserved time in the second scenario can make a higher travel cost of all alternative paths than in the first scenario. The travel cost of bus direct paths in the second scenario is higher than that of

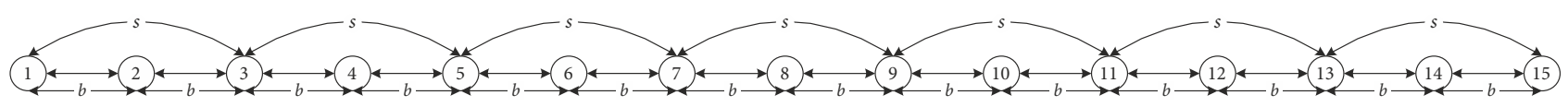

Figure 2. Bus and subway corridor 
Table 2. Potential demand matrix in transit corridor $[\mathrm{pax} / \mathrm{h}]$

\begin{tabular}{|c|c|c|c|c|c|c|c|c|c|c|c|c|c|c|c|}
\hline D & 1 & 2 & 3 & 4 & 5 & 6 & 7 & 8 & 9 & 10 & 11 & 12 & 13 & 14 & 15 \\
\hline 1 & 0 & 419 & 1786 & 236 & 1448 & 223 & 1474 & 198 & 1465 & 165 & 1497 & 164 & 1601 & 132 & 1575 \\
\hline 2 & 300 & 0 & 272 & 480 & 426 & 350 & 376 & 454 & 552 & 312 & 384 & 250 & 232 & 240 & 262 \\
\hline 3 & 1150 & 349 & 0 & 521 & 1799 & 258 & 1955 & 248 & 3074 & 182 & 2522 & 176 & 2159 & 89 & 3163 \\
\hline 4 & 212 & 225 & 406 & 0 & 412 & 300 & 292 & 279 & 283 & 261 & 288 & 250 & 167 & 190 & 160 \\
\hline 5 & 1946 & 275 & 2879 & 332 & 0 & 229 & 1390 & 267 & 1579 & 213 & 1272 & 206 & 1427 & 181 & 334 \\
\hline 6 & 161 & 141 & 300 & 335 & 160 & 0 & 214 & 200 & 216 & 235 & 246 & 250 & 222 & 200 & 210 \\
\hline 7 & 1456 & 171 & 2280 & 261 & 1201 & 193 & 0 & 215 & 1393 & 231 & 1546 & 234 & 1505 & 272 & 1564 \\
\hline 8 & 100 & 170 & 150 & 132 & 200 & 210 & 189 & 0 & 211 & 160 & 194 & 200 & 267 & 220 & 236 \\
\hline 9 & 2233 & 419 & 2210 & 236 & 1323 & 223 & 1345 & 198 & 0 & 204 & 1183 & 230 & 1451 & 218 & 1638 \\
\hline 10 & 340 & 358 & 286 & 440 & 466 & 350 & 379 & 503 & 386 & 0 & 356 & 310 & 251 & 286 & 295 \\
\hline 11 & 1584 & 218 & 2959 & 521 & 1774 & 258 & 1452 & 248 & 1322 & 221 & 0 & 271 & 1147 & 222 & 1414 \\
\hline 12 & 250 & 300 & 312 & 352 & 409 & 296 & 296 & 281 & 262 & 361 & 271 & 0 & 260 & 357 & 351 \\
\hline 13 & 1575 & 151 & 2286 & 317 & 1448 & 229 & 1484 & 267 & 1650 & 261 & 1318 & 295 & 0 & 362 & 1604 \\
\hline 14 & 160 & 182 & 260 & 262 & 200 & 200 & 173 & 208 & 162 & 190 & 170 & 165 & 162 & 0 & 180 \\
\hline 15 & 1645 & 135 & 1932 & 141 & 1550 & 205 & 1284 & 215 & 1140 & 205 & 1276 & 152 & 1272 & 201 & 0 \\
\hline
\end{tabular}

Table 3. Values of parameter

\begin{tabular}{|l|c|c|c|c|c|c|c|c|c|c|c|c|c|c|c|}
\hline Parameter & $\alpha_{1}$ & $\alpha_{2}$ & $\alpha_{3}$ & $\alpha_{4}$ & $\alpha_{5}$ & $\gamma_{b}$ & $\gamma_{s}$ & $\beta$ & $\theta$ & $\rho_{b}$ & $\rho_{s}$ & $\eta_{b}^{0}$ & $\eta_{b}^{1}$ & $\eta_{s}^{0}$ & $\eta_{s}^{1}$ \\
\hline Value & 1.2 & 2.0 & 1.0 & 0.125 & 0.8 & 0.5 & 0.5 & 0.6 & 3.5 & 1.2 & 1.1 & 0.5 & 0.1 & 0.1 & 0.02 \\
\hline
\end{tabular}

Table 4. Characteristics of bus and subway services

\begin{tabular}{|l|c|c|}
\hline \multicolumn{1}{|c|}{ Mode } & Bus & Subway \\
\hline Vehicle capacity $k_{m}[\mathrm{pax} / \mathrm{veh}]$ & 120 & 600 \\
\hline Operating cost $C_{m}[¥ /$ veh-h] & 80 & 900 \\
\hline Average operating speed $V_{m}[\mathrm{~km} / \mathrm{h}]$ & 10 & 30 \\
\hline Frequency $f_{m}[\mathrm{veh} / \mathrm{h}]$ & 60 & 20 \\
\hline
\end{tabular}

transfer paths or subway direct paths, which results in a lower passenger demand share on bus direct paths, a higher passenger demand share on subway direct paths and the same share on the two transfer paths in the second scenario comparing with the first scenario. Furthermore, the effects of on the reserved time also lead to a lower optimal fare of bus service and the same optimal fare of subway service in the third scenario comparing with the optimal fares in the first and second scenarios. Considering the second and third scenarios, the relevant time components of all alternative paths are the same in the two scenarios. In the SUE, the reduced fare components of travel cost of bus direct paths and transfer paths must be equilibrated by the increased in-vehicle crowding discomfort, which can be evidenced by the increased passenger demand shares of both bus direct paths (2.2\%) and transfer paths (1.3\%) in the third scenario comparing with the second scenario. Therefore, Table 5 indicates that the reserved trip time for transit service varies inversely with the optimal fare of service. In other words, the optimal fare of a transit service is lower if the service is disturbed by uncertain factors more often.
Table 5. Results of first experiment

\begin{tabular}{|l|l|c|c|c|c|c|}
\hline \multirow{2}{*}{ Scenario } & \multirow{2}{*}{$\begin{array}{c}\text { Reserved } \\
\text { time }\end{array}$} & \multicolumn{2}{|c|}{ Fare $p_{m}[¥]$} & \multicolumn{2}{|c|}{$\begin{array}{c}\text { Direct share } \\
{[\%]}\end{array}$} & \multirow{2}{*}{$\begin{array}{c}\text { Transfer } \\
\text { share } \\
{[\%]}\end{array}$} \\
\cline { 3 - 6 } & & Bus & Subway & Bus & Subway & \\
\hline First & $\begin{array}{l}\text { unconsi- } \\
\text { dered }\end{array}$ & 1.0 & 2.4 & 42.6 & 44.6 & 12.8 \\
\hline Second & considered & 1.0 & 2.4 & 40.6 & 46.6 & 12.8 \\
\hline Third & considered & 0.6 & 2.4 & 42.8 & 43.1 & 14.1 \\
\hline
\end{tabular}

\subsubsection{Effects of transfer discounts}

We now look at the benefit of transfer discounts to the social welfare, and the effects of transfer discounts on passenger demand shares among all alternative. Still type one of O-D pairs in Table 2 are used for this investigation. For this purpose, it is also necessary to consider three scenarios, which can be described as follows:

- first scenario: optimize fares of bus and subway services with original transfer cost (i.e. transfer discount $\lambda_{m}$ equals $\left.100 \%\right)$;

- second scenario: optimize transfer discounts of bus and subway services with the given fares inherited from the first scenario;

- third scenario: optimize fares and transfer discounts of bus and subway services integrally.

The results from the second experiment are displayed in Table 6. Considering the first and second scenarios, the fares of bus and subway services are the same. With the optimization of transfer discounts in the second scenario, the relevant time and fare components of travel cost of bus 
and subway direct paths are fixed, but the fare components of travel cost of bus-subway transfer paths are changed due to the optimal transfer discount. Thus, in SUE, the reduced fare components of travel cost of transfer paths must be equilibrated by the increased in-vehicle crowding discomfort in travel cost of these paths. Correspondingly, the in-vehicle crowding discomfort is reduced. The changes can be illustrated by the increased passenger demand shares of transfer paths and the reduced passenger demand shares of direct paths in the second scenario comparing with the first scenario. In addition, the optimization of transfer discounts can also benefit the passenger demand and social welfare, which increase by $84 \mathrm{pax} / \mathrm{h}$ and $115 ¥ / \mathrm{h}$, respectively.

Comparing with the fares and transfer discounts in the first and second scenarios, the optimal fares in the third scenario are increased, and the resulting transfer discounts are reduced. Based on the fixed relevant time components of travel cost in all three scenarios, the increased relevant fare components of travel cost of direct paths and reduced fare components (fares and transfer pricings) of travel cost of transfer paths must make the passenger demand share of direct paths reduced and the share of transfer paths increased in the third scenario. Furthermore, the changes of passenger demand share of bus direct paths are more than those of subway direct paths due to the more increased fares of subway. For example, the optimal fare of subway service in the third scenario increases by $0.3 ¥$, and the fare of bus service only increases by $0.1 ¥$ comparing with that in the second scenario, which results in a lager reduced value (3.5\%) in the passenger demand share of subway direct paths and a smaller reduced value $(0.1 \%)$ in the passenger demand share of bus direct paths, respectively. Obviously, the social welfare in the third scenario can benefit from the integrated optimization of fares and transfer discounts in spite of the reduced passenger demand.
Table 7 displays the detailed changes in the passenger demand share of transfer paths in the given demand matrix. The transfer share in the first and third scenarios are shown as example. With the increasing travel length, the advantage of transfer paths over bus direct paths rises in terms of travel time. Therefore, the passenger demand shares of transfer paths increase with the travel length in both scenarios. With the same travel length, the passenger demand shares of transfer paths in the third scenario are higher than in the first scenario in term of the benefit of transfer discounts that are applied in the third scenario. The difference in demand share between the two scenarios can range from 5 to $8 \%$, which shows that it is essential to adopt transfer discounts in an integrated transit corridor with aim to encourage more transfers.

\subsubsection{Effects of added passenger $\mathrm{O}-\mathrm{D}$ pairs considering the different stop distances of two services}

We explore the interplay of passenger behavior among different $\mathrm{O}-\mathrm{D}$ pair types, and further effects on the optimization of fare structure in the third experiment. For this purpose, we also look at three scenarios, which are designed as follows:

- first scenario: optimize fare structure without difference in stop distance, which means only type one $\mathrm{O}-\mathrm{D}$ pairs (dash area in Table 2) are considered;

- second scenario: capture passenger demand shares among different paths considering the difference in stop distance between bus and subway services based on the fare structure inherited from the first scenario;

- third scenario: optimize fares and transfer discounts of bus and subway services considering the difference in stop distance between the two services.

The results from the third experiment are displayed in Table 8. Comparing with those from the first scenario, the added $\mathrm{O}-\mathrm{D}$ pairs in the second scenario, namely, type

Table 6. Results of second experiment

\begin{tabular}{|c|c|c|c|c|c|c|c|c|c|}
\hline \multirow{2}{*}{ Scenario } & \multicolumn{2}{|c|}{ Fare $p_{m}[¥]$} & \multicolumn{2}{|c|}{ Discount $\lambda_{m}[\%]$} & \multicolumn{2}{|c|}{ Direct share [\%] } & \multirow{2}{*}{$\begin{array}{l}\text { Transfer } \\
\text { share [\%] }\end{array}$} & \multirow{2}{*}{$\begin{array}{l}\text { Demand } \\
{[\mathrm{pax} / \mathrm{h}]}\end{array}$} & \multirow{2}{*}{$\begin{array}{c}S W \\
{[¥ / \mathrm{h}]}\end{array}$} \\
\hline & Bus & Subway & Bus & Subway & Bus & Subway & & & \\
\hline First & 0.6 & 2.4 & 100 & 100 & 42.8 & 43.1 & 14.1 & 68700 & 1015539 \\
\hline Second & 0.6 & 2.4 & 100 & 87 & 42.7 & 42.5 & 14.9 & 68784 & 1015654 \\
\hline Third & 0.7 & 2.7 & 30 & 50 & 42.6 & 39.0 & 18.4 & 68375 & 1016110 \\
\hline
\end{tabular}

Table 7. Changes in passenger demand share of transfer path [\%] in second experiment: third scenario/first scenario

\begin{tabular}{|c|c|c|c|c|c|c|c|c|c|}
\hline $\mathrm{D}$ & $\mathrm{O}$ & 1 & 3 & 5 & 7 & 9 & 11 & 13 & 15 \\
\hline 1 & - & $0 / 0$ & $21 / 16$ & $23 / 18$ & $26 / 20$ & $30 / 23$ & $33 / 25$ & $35 / 27$ \\
\hline 3 & $0 / 0$ & - & $0 / 0$ & $21 / 16$ & $23 / 18$ & $26 / 20$ & $30 / 23$ & $33 / 25$ \\
\hline 5 & $21 / 16$ & $0 / 0$ & - & $0 / 0$ & $21 / 16$ & $23 / 18$ & $26 / 20$ & $30 / 23$ \\
\hline 7 & $23 / 18$ & $21 / 16$ & $0 / 0$ & - & $0 / 0$ & $21 / 16$ & $23 / 18$ & $26 / 20$ \\
\hline 9 & $27 / 20$ & $23 / 18$ & $21 / 16$ & $0 / 0$ & - & $0 / 0$ & $21 / 16$ & $23 / 18$ \\
\hline 11 & $30 / 23$ & $26 / 20$ & $23 / 17$ & $21 / 16$ & $0 / 0$ & - & $0 / 0$ & $21 / 16$ \\
\hline 13 & $33 / 25$ & $30 / 23$ & $26 / 20$ & $23 / 18$ & $21 / 16$ & $0 / 0$ & - & $0 / 0$ \\
\hline 15 & $35 / 27$ & $32 / 25$ & $30 / 23$ & $26 / 20$ & $23 / 18$ & $21 / 16$ & $0 / 0$ & - \\
\hline
\end{tabular}


two and three O-D pairs cause in-vehicle overcrowding of both services, which are illuminated by increased highest loading degree in bus and subway lines (increased by 2.9 and $4.1 \%$, respectively). The effects of the added O-D pairs on passenger behavior of three types and passenger demand shares among several alternative paths are elaborated in the following analysis.

Passengers of type two O-D pairs must complete their trips by bus direct paths. The travel costs of bus paths increase with passengers' travel length. Thus, the travel ratio of type one O-D pairs decreases with travel length, e.g. travel ratio for O-D pairs $(2,1),(2,4),(2,6),(2,8)$, $(2,10),(2,12)$ and $(2,14)$ in Figure 3.

Passengers of type three O-D pairs can complete their trips by bus direct paths and transfer paths. The travel ratio of type one O-D pairs decreases with travel length. According to the assumption of transfer path in Section 1.1, the superiority of transfer paths increases with travel length comparing with bus direct paths, which result in an increasing transfer share and a decreasing bus direct share with the increasing travel length. Figure 4 shows the change in travel ratio, bus direct share and transfer share for type two O-D pairs $(1,4),(1,6),(1,8),(1,10),(1,12)$ and $(1,14)$ as example.

Types two and three O-D pairs added in the second scenario increase the passenger flow on bus line directly, which must lead to more in-vehicle crowding discomfort in the travel cost of bus direct paths. Thus, type one O-D pairs in the second scenario have a lower bus direct share comparing with the first scenario. More passengers of this type O-D pairs are likely to choose subway direct paths. Figures 5 and 6 show the different path shares for type one $\mathrm{O}-\mathrm{D}$ pairs in the first and second scenarios, respectively. This shows that the subway direct path share has a significant rise, which is consistent with the results in Table 8 in which the passenger flow of subway direct path increases by $1405 \mathrm{pax} / \mathrm{h}$. In addition, comparing with the first sce- nario, the increased passenger flow of bus direct paths in the second scenario results from the added passenger demand of types two and three, and the increased transfer paths share in the second scenario is caused by the added transfer flow of type three and transfer flow of type one $\mathrm{O}-\mathrm{D}$ pairs based on the decreased travel ratio (from 73.4 to $60.3 \%$ ). Therefore, considering the difference in stop distance between bus and subway lines, the passenger behavior of added O-D pairs interacts with type one O-D pairs significantly, which imply that the difference in stop distance can not be ignored, otherwise the overcrowding may appear in transit vehicles.

Furthermore, the overcrowding in transit vehicles in the second scenario also may forebear from the higher optimal fares in the third scenario. As shown in Table 8, subject to the capacity of transit vehicles, the fares of bus and subway services rise to a level similar to transfer discounts. Comparing with the second scenario, the higher fares not only result in a lower travel ratio, but also change the passenger demand shares among different paths. For example, with the close gap of fares between the two services, the superiority of cheaper fares for bus direct paths falls down comparing with subway direct paths, which results in a decreased passenger flow in bus direct paths and increased passenger flow in subway direct paths, as shown in Table 8.

\subsubsection{Sensitivity analysis of passenger demand of added $\mathrm{O}-\mathrm{D}$ pairs}

We carry out a sensitivity analysis of the impacts on optimal fares and transfer discounts of bus and subway services of varying the passenger demands of added O-D pairs (types two and three $\mathrm{O}-\mathrm{D}$ pairs). The passenger demands of added O-D pairs in Table 2 are marked as $Q^{*}$. We optimize the fare structure with the variable $Q^{*}$, i.e. 80,60 and $40 \%$ of $Q^{*}$. The results are displayed in Table 9.

Table 8. Results of third experiment

\begin{tabular}{|c|c|c|c|c|c|c|c|c|c|c|}
\hline \multirow{2}{*}{ Scenario } & \multicolumn{2}{|c|}{$\begin{array}{l}\text { Fare } p_{m} \\
\quad[¥]\end{array}$} & \multicolumn{2}{|c|}{$\begin{array}{c}\text { Discount } \lambda_{m} \\
{[\%]}\end{array}$} & \multicolumn{2}{|c|}{$\begin{array}{l}\text { Passenger flow } \\
{[\mathrm{pax} / \mathrm{h}]}\end{array}$} & \multirow{2}{*}{$\begin{array}{c}\text { Transfer share } \\
{[\%]}\end{array}$} & \multicolumn{2}{|c|}{$\begin{array}{l}\text { Highest loading } \\
\text { degree [\%] }\end{array}$} & \multirow{2}{*}{$\begin{array}{c}\text { Travel ratio } \\
{[\%]}\end{array}$} \\
\hline & Subway & Bus & Subway & Bus & Subway & Bus & & Subway & Bus & \\
\hline First & 2.7 & 0.7 & 50 & 30 & 26687 & 29126 & 18.4 & 100.1 & 98.2 & 73.4 \\
\hline Second & 2.7 & 0.7 & 50 & 30 & 28092 & 36898 & 18.9 & 104.2 & 101.1 & 60.3 \\
\hline Third & 5.3 & 5.3 & 50 & 50 & 32718 & 31656 & 9.5 & 100.1 & 100.0 & 53.6 \\
\hline
\end{tabular}

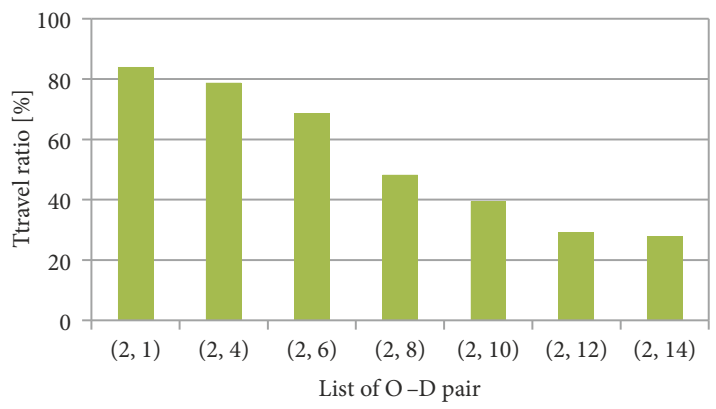

Figure 3. Travel ratio for passengers of type two O-D pairs in second scenario

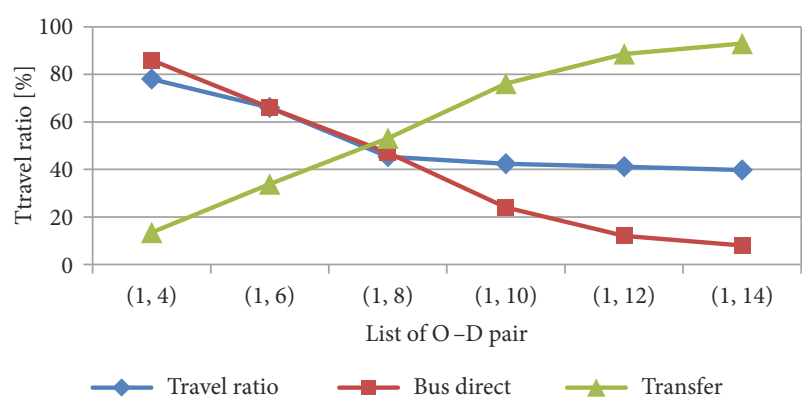

Figure 4. Travel ratio and paths shares for type three O-D pairs in second scenario 


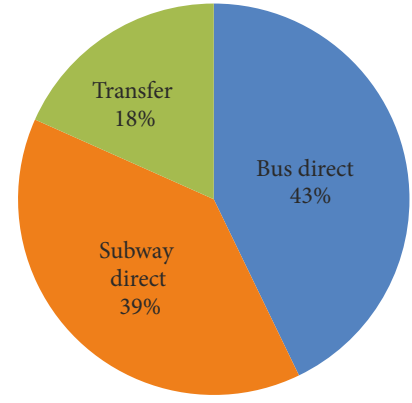

Figure 5. Paths share for type one O-D pairs in first scenario

As shown in Table 9, as the passenger demand of added O-D pairs decreases, both the fares of the two services and the transfer discount fall whereas the difference in fare between subway and bus services rises. Therefore, the passenger demand of added O-D pairs may have an indirect impact on the fare and transfer discount of the subway service and directly affect the bus service.

Table 9. Results of sensitivity analysis

\begin{tabular}{|c|c|c|c|c|}
\hline \multirow{2}{*}{$Q^{*}$} & \multicolumn{2}{|c|}{ Fare $p_{m}[¥]$} & \multicolumn{2}{c|}{ Discount $\lambda_{m}[\%]$} \\
\cline { 2 - 5 } & Subway & Bus & Subway & Bus \\
\hline $80 \%$ & 4.7 & 4.5 & 60 & 50 \\
\hline $60 \%$ & 4.6 & 4.0 & 40 & 30 \\
\hline $40 \%$ & 4.0 & 3.0 & 40 & 20 \\
\hline
\end{tabular}

\section{Concluding remarks}

This paper has formulated a bi-level program to optimize the fare structure (including fares and transfer discounts) along a bus-subway corridor with an objective of maximizing the social welfare. The two important factors have been considered in this optimization problem. One is the uncertainty in travel time of mode, which is due to the unstable operational environment of transit modes and/or random factors. The degree of travel time uncertainty differs from mode to mode even though they run along the same corridor. Thus, the influence of uncertainty in travel time on passenger behavior is formulated as a reserved time in the optimization model proposed in this paper, which is one of the intellectual merits of this work.

The second factor is the difference in stop spacing between bus and subway services. In a bus-subway corridor with different stop distances of the two services, passengers complete their trips by different sets of alternative paths. For example, some O-D pairs can be served by direct bus paths, some by direct subway paths and some by those paths combining subway and bus services. Thus, $\mathrm{O}-\mathrm{D}$ pairs in such a corridor can be classified into three types. Type one O-D pairs are consistent with the travel groups in the existing literature, which does not focus on the difference in stop spacing between different mode services, and types two and three O-D pairs proposed in this

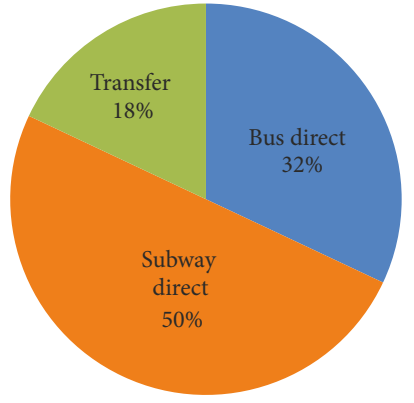

Figure 6. Paths share for type one O-D pairs in second scenario

paper are the newly added travel groups due to the stop spacing difference issue.

Numerical experiments have been designed to explore the four issues: effects of reserved time on optimal fares, effects of transfer discounts, effects of difference in stop distance between bus and subway services and sensitivity analysis of passenger demand of the two newly added types of O-D pairs. Here is a list of new findings:

- the consideration of reserved time in a trip has a significant impact on the optimal fares of bus and subway services. If a service is more easily disturbed due to uncertain factors then the more reserved time shall be required in a trip by this service and the optimal fare of it will be lower;

- the introduction of transfer discounts of corridor transit services is of great benefit to the social welfare and transfers;

- type two O-D pairs have to choose the direct bus paths while the type three O-D pairs are likely to choose bus direct paths for short distance travel and mode-combined paths for long distance travel. The newly-added passenger $\mathrm{O}-\mathrm{D}$ pairs share a certain ratio of capacity of bus service, which leads to a lower bus direct share of type one O-D pairs comparing with no consideration of the difference in stop spacing between bus and subway services. More type one passengers are likely to choose subway direct paths. In addition, these changes result in an increased bus and subway fare because of the increased congestion in bus and subway lines in the end. The observed passenger behavior indicates that it is of utmost importance to consider the difference in stop spacing between bus and subway services while optimizing public transit fare structures for different mode services along a corridor;

- the newly added types of O-D pairs have a direct impact on the optimal fare of bus service and have an indirect impact on the fare of subway service.

The major challenge in this investigation is how to assign the reasonable values of parameters accurately when we implement the case study on real-life road networks. Some stated preference surveys are needed, and it is necessary to consider the heterogeneity of passengers. Although these difficulties exist, the essential ideas of the paper are still valuable to the optimization of fare structure for a bus-subway corridor. 
A possible extension of this research would be to divide passengers into groups with different trip purposes and ages because the desired travel comfort, waiting time and fare cost vary for different groups. An ongoing piece of our research is to design an applicable fare structure for groups with different purposes and/or ages along a bussubway corridor.

\section{Acknowledgements}

This research is mainly supported by the Lloyd's Register Foundation, which supports the advancement of engineering-related education, and funds research and development that enhances safety of life at sea, on land and in the air.

It is also partly supported by the National Natural Science Foundation of China (Grant No 71431003).

The support of the two agencies/organizations is gratefully acknowledged.

\section{References}

Avineri, E. 2006. The effect of reference point on stochastic network equilibrium, Transportation Science 40(4): 409-420. https://doi.org/10.1287/trsc.1060.0158

Ben-Akiva, M.; Lerman, S. R. 1985. Discrete Choice Analysis: Theory and Application to Travel Demand. MIT Press. $412 \mathrm{p}$.

Borndörfer, R.; Karbstein, M.; Pfetsch, M. E. 2012. Models for fare planning in public transport, Discrete Applied Mathematics 160(18): 2591-2605. https://doi.org/10.1016/j.dam.2012.02.027

Chen, B. Y.; Shi, C.; Zhang, J.; Lam, W. H. K.; Li, Q.; Xiang, S. 2017. Most reliable path-finding algorithm for maximizing on-time arrival probability, Transportmetrica B: Transport Dynamics 5(3): 248-264. https://doi.org/10.1080/21680566.2016.1169953

De Palma, A.; Kilani, M.; Proost, S. 2015. Discomfort in mass transit and its implication for scheduling and pricing, Transportation Research Part B: Methodological 71: 1-18. https://doi.org/10.1016/j.trb.2014.10.001

Ehrgott, M.; Wang, J. Y. T.; Watling, D. P. 2015. On multi-objective stochastic user equilibrium, Transportation Research Part B: Methodological 81: 704-717.

https://doi.org/10.1016/j.trb.2015.06.013

Evans, A. 1987. A theoretical comparison of competition with other economic regimes for bus services, Journal of Transport Economics and Policy 21(1): 7-36.

Frejinger, E.; Bierlaire, M. 2007. Capturing correlation with subnetworks in route choice models, Transportation Research Part B: Methodological 41(3): 363-378.

https://doi.org/10.1016/j.trb.2006.06.003

Fujii, S.; Kitamura, R. 2004. Drivers' mental representation of travel time and departure time choice in uncertain traffic network conditions, Networks and Spatial Economics 4(3): 243256. https://doi.org/10.1023/B:NETS.0000039781.10517.3a

Glaister, S.; Collings, J. J. 1978. Maximisation of passenger miles in theory and practice, Journal of Transport Economics and Policy 12(3): 304-321.

Kaddoura, I.; Kickhöfer, B.; Neumann, A.; Tirachini, A. 2015. Agent-based optimisation of public transport supply and pricing: impacts of activity scheduling decisions and simulation randomness, Transportation 42(6): 1039-1061.

https://doi.org/10.1007/s11116-014-9533-6
Kocur, G.; Hendrickson, C. 1982. Design of local bus service with demand equilibration, Transportation Science 16(2): 149-170. https://doi.org/10.1287/trsc.16.2.149

Lam, W.; Morrall, J. 1982. Bus passenger walking distances and waiting times: a summer-winter comparison, Transportation Quarterly 36(3): 407-421.

Lam, W. H. K.; Zhou, J. 2000. Optimal fare structure for transit networks with elastic demand, Transportation Research Record: Journal of the Transportation Research Board 1733: 8-14. https://doi.org/10.3141/1733-02

Li, Z.-C.; Lam, W. H. K.; Wong, S. C. 2009a. Optimization of a bus and rail transit system with feeder bus services under different market regimes, in W. H. K. Lam, S. C. Wong, H. K. Lo (Eds.). Transportation and Traffic Theory 2009: Golden Jubilee: 495-516. https://doi.org/10.1007/978-1-4419-0820-9_25

Li, Z.-C.; Lam, W. H. K.; Wong, S. C. 2009b. The optimal transit fare structure under different market regimes with uncertainty in the network, Networks and Spatial Economics 9(2): 191-216. https://doi.org/10.1007/s11067-007-9058-Z

Liu, B.-Z.; Ge, Y.-E.; Cao, K.; Jiang, X.; Meng, L.; Liu, D.; Gao, Y. 2017. Optimizing a desirable fare structure for a bus-subway corridor, Plos One 12(10): e0184815.

https://doi.org/10.1371/journal.pone.0184815

Lu, X.-S.; Liu, T.-L.; Huang, H.-J. 2015. Pricing and mode choice based on nested logit model with trip-chain costs, Transport Policy 44: 76-88. https://doi.org/10.1016/j.tranpol.2015.06.014

Nash, C. A. 1978. Management objectives, fares and service levels in bus transport, Journal of Transport Economics and Policy 12(1): 70-85.

Noland, R. B.; Polak, J. W. 2002. Travel time variability: a review of theoretical and empirical issues, Transport Reviews 22(1): 39-54. https://doi.org/10.1080/01441640010022456

Oppenheim, N. 1995. Urban Travel Demand Modeling: from Individual Choices to General Equilibrium. Wiley, New York. $480 \mathrm{p}$.

Ramming, M. C. 2002. Network Knowledge and Route Choice. PhD Dissertation. Massachusetts Institute of Technology, US. 394 p. Available from Internet: https://dspace.mit.edu/handle/1721.1/49797

Shao, H.; Lam, W. H. K.; Meng, Q.; Tam, M. L. 2006. Demanddriven traffic assignment problem based on travel time reliability, Transportation Research Record: Journal of the Transportation Research Board 1985: 220-230. https://doi.org/10.1177/0361198106198500124

Sheffi, Y. 1985. Urban Transportation Networks: Equilibrium Analysis with Mathematical Programming Methods. Prentice Hall. 399 p.

Spiess, H.; Florian, M. 1989. Optimal strategies: a new assignment model for transit networks, Transportation Research Part B: Methodological 23(2): 83-102.

https://doi.org/10.1016/0191-2615(89)90034-9

Tang, C.; Ceder, A.; Ge, Y.-E. 2017. Integrated optimization of bus line fare and operational strategies using elastic demand, Journal of Advanced Transportation 2017: 7058789. https://doi.org/10.1155/2017/7058789

Tang, C.; Ceder, A.; Zhao, S.; Ge, Y.-E. 2016. Determining optimal strategies for single-line bus operation by means of smartphone demand data, Transportation Research Record: Journal of the Transportation Research Board 2539: 130-139. https://doi.org/10.3141/2539-15

Tang, C.; Ge, Y.-E.; Lam, W. H. K. 2019. Optimizing limited-stop bus services along a public transit corridor with a differential fare structure, Transport 34(4): 476-489.

https://doi.org/10.3846/transport.2019.11235 
Tirachini, A.; Hensher, D. A. 2011. Bus congestion, optimal infrastructure investment and the choice of a fare collection system in dedicated bus corridors, Transportation Research Part B: Methodological 45(5): 828-844.

https://doi.org/10.1016/j.trb.2011.02.006

Tirachini, A.; Hensher, D. A.; Rose, J. M. 2014. Multimodal pricing and optimal design of urban public transport: the interplay between traffic congestion and bus crowding, Transportation Research Part B: Methodological 61: 33-54. https://doi.org/10.1016/j.trb.2014.01.003

Tong, C. O.; Wong, S. C. 1999. A stochastic transit assignment model using a dynamic schedule-based network, Transportation Research Part B: Methodological 33(2): 107-121. https://doi.org/10.1016/S0191-2615(98)00030-7

Wang, W.; Sun, H.; Wang, Z.; Wu, J. 2014. Optimal transit fare in a bimodal network under demand uncertainty and bounded rationality, Journal of Advanced Transportation 48(8): 957-973. https://doi.org/10.1002/atr.1238

Watling, D. 2006. User equilibrium traffic network assignment with stochastic travel times and late arrival penalty, European Journal of Operational Research 175(3): 1539-1556. https://doi.org/10.1016/j.ejor.2005.02.039

Williams, H. C. W. L. 1977. On the formation of travel demand models and economic evaluation measures of user benefit, Environment and Planning A: Economy and Space 9(3): 285344. https://doi.org/10.1068/a090285

Yang, H.; Kin, W. K. 2000. Modeling bus service under competition and regulation, Journal of Transportation Engineering 126(5): 419-425.

https://doi.org/10.1061/(ASCE)0733-947X(2000)126:5(419)

Yao, B.; Hu, P.; Lu, X.; Gao, J.; Zhang, M. 2014. Transit network design based on travel time reliability, Transportation Research Part C: Emerging Technologies 43: 233-248. https://doi.org/10.1016/j.trc.2013.12.005

Yao, J.; Shi, F.; An, S.; Wang, J. 2015. Evaluation of exclusive bus lanes in a bi-modal degradable road network, Transportation Research Part C: Emerging Technologies 60: 36-51. https://doi.org/10.1016/j.trc.2015.08.005

Zhou, J.; Lam, W. H. K.; Heydecker, B. G. 2005. The generalized Nash equilibrium model for oligopolistic transit market with elastic demand, Transportation Research Part B: Methodological 39(6): 519-544. https://doi.org/10.1016/j.trb.2004.07.003 